\title{
Findings from the Yearbook 2010 Section on Decision Support Systems
}

\author{
P. Ruch, Section Editor for the IMIA Yearbook Section on Decision Support Systems \\ University of Applied Sciences Geneva, Dept. of Information and Library Sciences, Geneva, Switzerland
}

\section{Introduction}

The five papers selected this year, clearly address different aspects of decision support. De Jong et al.'s paper reports on the observed impact of decision support systems on physicians practice patterns in the Netherland. Wright et al.'s uses a set of interviews to deliver a comparative survey of decision-support systems in commercially available Electronic Health Records. Ernesater et al. report on a qualitative study to assess the acceptance and perception of the telenurse decision support system by professionals. Goud et al report on a trial trying to assess the impact of computer-aided decision systems to improve rehabilitation of patient suffering from cardiac pathologies. Finally, Kohler et al. proposes an application to help diagnosis of genetic disorders using the Human Phenotype Ontology.

\section{Best Paper Selection}

The best paper selection of articles for the section on decision-support systems in the IMIA Yearbook 2010 follows the tradition of previous Yearbooks in presenting excellent research on methods used for the implementation of computer tools to help healthcare agents to make better decision at bedside, as well as at population levels. Four of the selected papers are directly related to the evaluation of instruments likely to improve concordance with guidelines and quality of care in general. A fifth paper attempts to derive a differential diagnosis application to help decision-making based on signs and symptoms of genetic pathologies, somehow similar to diagnosis association tools as described in $[1,2]$. The objectives are to reduce errors, adverse effects, and costs in the care and/or diagnosis process $[3,4]$. Table 1 presents the selected papers. A brief content summary of the selected best papers can be found in the appendix of this report.

\section{Conclusions and Outlook}

The best paper selection for the Yearbook section on decision support systems can by no means reflect the broadness of a field that is intrinsically heterogeneous. The selected papers, however, shed light on some special aspects deserving particular attention as they concern key methodological questions for the future of the field. In particular two papers are reporting on experiments which are also registered clinical trials. It is thus questioned whether DSS should be considered as therapeutic target like any other medicinal drugs or professional intervention, such as surgery of psychology. While decision-support system seems to effectively support experts in their daily practices, a specific regulation framework might be needed in order not to hinder exploratory and emerging initiatives in the field. Indeed, while recommendation systems are likely to significantly improve decision making, their promotion to the level of official ,treatment" can result in two critical drawbacks: 1. a lower acceptance by professionals, who would feel replaced by machines; 2. a legitimate rejection by patients, who do not want to be treated by computers. In this context human-mediation shall soon appear as a must for future success stories of computerized decision support systems. 


\section{Acknowledgement}

I greatly acknowledge the support of Martina Hutter and of the reviewers in the selection process of the IMIA Yearbook.

\section{References}

1. Pakhomov SV, Buntrock JD, Chute CG. Automating the assignment of diagnosis codes to patient encounters using example-based and machine learning techniques. J Am Med Inform Assoc 2006 Sep-Oct;13(5):516-25. Epub 2006 Jun 23.

2. Ruch P, Gobeill J, Tbahriti I, Geissbühler A . From episodes of care to diagnosis codes: automatic text categorization for medico-economic encoding.AMIA Annu Symp Proc 2008 Nov 6:636-40.

3. Kohn LT, Corrigan JM, Donaldson MS, editors. To Err Is Human: Building a Safer Health System. Institute of Medicine (U.S.) Committee on Quality of Health Care in America. Washington, DC: National Academy Press; 1999.

4. Han YY, Carcillo JA, Venkataraman ST, Clark RS, Watson RS, Nguyen TC, et al. Unexpected increased mortality after implementation of a commercially sold computerized physician order entry system. Pediatrics. 2005 Dec;116(6):1506-12. Erratum in: Pediatrics. 2006 Feb;117(2):59.

Correspondence to:

Prof. Dr. Patrick Ruch

University of Applied Sciences Genevo

Department of Library and Information Sciences

Geneva, Switzerland

Tel: +41 223881781

E-mail: patrick.ruch@hesge.ch

\section{Appendix: Content Summaries of Selected Best Papers for the IMIA Yearbook 2010, Section Decision Support Systems*}

\author{
de Jong JD, Groenewegen PP, Spreeuwenberg \\ P, Westert GP, de Bakker DH \\ Do decision support systems influence varia- \\ tion in prescription?
}

BMC Health Serv Res 2009 Jan 30;9:20
Changing behaviours by translating scientific evidence into daily practice is a challenging task. So far, all known strategies, such as educational and/or directive strategies, must still be regarded as partially successful. In this article the authors focus is on computer-aided decision support systems (DSSs). A subset of those systems aims at changing physicians' practice pattern, as opposed to interventions that aim at influencing knowledge in order to change behavior. The authors focus on exploring the impact of DSS to improve general practitioners' prescription agreement (GPs) METHODS: Data from the Second Dutch National Survey of General Practice (DNSGP2), are used. A total of 82 diagnoses, 749811 contacts, 133 physicians, and 85 practices, was included in the analyses. GPs using the DSS daily were compared to GPs who do not use the DSS. Two outcome measures were chosen: whether prescription was in accordance with the advice of the DSS or not, and a measure of concentration, the HerfindahlHirschman Index (HHI). It is observed that agreement with guidelines is improved for GPs, which are daily users of the DSS. More surprisingly, variation in Prescription, as measured by HHI was comparable for both groups of GPs. GPs using the DSS more often prescribe in accordance with the advice given in the DSS compared to GPs not using the DSS. However, prescription variation is the same for GPs using and for GPs not using a DSS. It is thus suggested that DSSs can be used to implement guidelines, but their impact on prescription variations is limited.

\section{Wright A, Sittig DF, Ash JS, Sharma S, Pang $\mathrm{JE}$, Middleton B}

Clinical decision support capabilities of commercially-available clinical information systems

\section{J Am Med Inform Assoc 2009 Sep- 0ct;16(5):637-44. Epub 2009 Jun 30}

Decision Supports Systems (DSS) embedded into Computerized Provider Order Entry (CPOE) and Electronic Health Records (EHR) provide additional value to both CPOE and EHRs. The authors aim at describing the results of a study of decision support capabilities in Certification Commission for Health Information Technology (CCHIT) certified electronic health record systems. A series of interviews with representatives of nine commercially available clinical information systems is conducted to analyse their characteristics against a 42 different clinical decision support features. Six of the nine evaluated systems matched all the applicable event-driven, actionoriented, real-time clinical decision support triggers required to successfully perform clinical decision support interventions. Nearly half (5) could access all the patient-specific data items identi-

Table 1 Best paper selection of articles for the IMIA Yearbook of Medical Informatics 2010 in the section 'Decision Support Systems'. The articles are listed in alphabetical order of the first author's surname.

Section

Decision Support Systems

- de Jong JD, Groenewegen PP, Spreeuwenberg P, Westert GP, de Bakker DH. Do decision support systems influence variation in prescription? BMC Health Serv Res 2009 Jan 30;9:20.

- Ernesäter A, Holmström I, Engström M. Telenurses' experiences of working with computerized decision support: supporting, inhibiting and quality improving. J Adv Nurs 2009 May;65(5):1074-83.

- Goud R, de Keizer NF, ter RietG, Wyatt JC, Hasman A, Hellemans IM, Peek N. Effect of guideline based computerised decision support on decision making of multidisciplinary teams: luster randomised trial in cardiac rehabilitation. BMJ 2009 Apr 27;338:b1440.

- Köhler S, Schulz MH, KrawitzP, Bauer S, Dökken S, OHtCE, Mundlos C, Horn D, Mundlos S, Robinson PN. Clinical diagnostics in human genetics with semantic similarity searches in ontologies. Am J Hum Genet 2009 0ct;85(4):457-64.

- Wright A, Sittig DF, Ash JS, Sharma S, Pang JE, Middleton B. Clinical decision support capabilities of commercially-available clinical information systems. J Am Med Inform Assoc 2009 Sep-0ct;16(5):637-44. Epub 2009 Jun 30. 
fied as necessary. Six of the nine systems supported all the intervention types required to allow clinical information systems to tailor their interventions based on the severity of the clinical situation. Only a unique system was able to deliver all key features to allowing physicians to take action directly from within the alert. The best system in our analysis had only a unique missing feature. In contrast, the less effective had eighteen missing features. We can thus observe the important variability of capability among commercially available systems with respect to DSS. It is suggested that such a contrasted observation should be monitored by potential users of clinical information systems, as well as developers, vendors of clinical information systems, and finally, certification bodies.

\section{Ernesäter A, Holmström I, Engström M Telenurses' experiences of working with computerized decision support: supporting, inhibiting and quality improving}

\section{J Adv Nurs 2009 May;65(5):1074-83}

The authors reports on a study, whose main aim is to describe telenurses' experiences of working with computerized decision support systems, with a focus on assessing the impact of such a system on nurses practice patterns. Telenursing is a fast growing service in many Western countries, and centralization of telenursing services is now observed in several countries such as Sweden. In parallel, the use of computer-assisted decision support has recently increased. Therefore, we decided to study via interviews the perception of eight Registered Nurses from three telephone call centres, who were using computerized decision support instruments. Collected data were then analysed using qualitative content analysis along the following dimensions: decision support system were to be judged as supporting, inhibiting and quality improving. Further focusing on two of the dimensions - ,supporting ${ }^{6}$ and , inhibiting ${ }^{6}$ - specific sub-features were identified: being strengthened, but simultaneously controlled and inhibited. Computer-assisted decision support tools were described as: simplifying their work, complementing their knowledge, providing them more security and credibility. As for negative outcomes, they also reported that decision support systems (DSS) are incomplete, inconsistent with their own opinions and controlling. The third category addressed organizational issues: the DSS were seen as ensuring better quality telenursing. Altogether, it is concluded that positive perception overcomes negative ones and nurses preferred working with it. Nevertheless, telenurses experienced computerized decision support as both supporting and inhibiting. Finally, it is also felt like computerized decision support systems are complementary but cannot replace telenurses' knowledge and competence.

\section{Goud R, de Keizer NF, ter Riet G, Wyatt JC, Hasman A, Hellemans IM, Peek N \\ Effect of guideline based computerised decision support on decision making of multidisciplinary teams: cluster randomised trial in cardiac reha- bilitation \\ BMJ 2009 Apr 27;338:b1440}

This paper aims at assessing how computer-assisted decision support can positively affect compliance with clinical practice guidelines in multidisciplinary teams. A multi-site randomised trial has been set up to cover multidisciplinary cardiac rehabilitation teams in Dutch centres and heir cardiac rehabilitation patients. Each team was provided with the same electronic patient record system with or without additional guideline based decision support. Concordance with legacy recommendations were assessed for two standard rehabilitation treatments-exercise and education therapy-and for two new but evidence based rehabilitation treatments-relaxation and lifestyle change therapy. From a methodological perceptive, intracluster correlations were adjusted for confounders such as patient's age, sex, and indication for cardiac rehabilitation and for type and volume of centre. Data from 21 centres, for a total of 2787 patients, were collected. The impact of guidelines results in a concordance increase of $7.9 \%$ for exercise therapy, for education therapy, it improves by $25.7 \%$, while for relaxation therapy it improves by $25.5 \%$. Finally, the concordance for lifestyle change therapy results in a $+3.2 \%$ improvement. It is also observed that computerised decision support reduces overtreatment and undertreatment. The authors conclude that decision supports systems using clinical practice guidelines can be effective instruments to significantly improve concordance with guidelines.

\section{Köhler S, Schulz MH, Krawitz P, Bauer S, Dölken S, Ott CE, Mundlos C, Horn D, Mundlos S, Robinson PN \\ Clinical diagnostics in human genetics with semantic similarity searches in ontologies \\ Am J Hum Genet 2009 0ct;85(4):457-64}

Differential diagnosis instruments can help physicians to identify candidate diseases that best explain a set of clinical features and to highlight those specific symptoms or diagnosis procedures likely to reduce the span of possible diagnosis. This process can be further complicated by the fact that discriminating features can have varying levels of specificity. In addition, several features can be observed, which are not directly related to the disease itself, but rather to co-morbidities. Depending on the experience of the professional and the set of laboratory and diagnosis tests available in the healthcare environment, clinical signs and symptoms may be described in greater or lesser detail. The authors have designed a specific semantic similarity metrics to measure phenotypic similarity between user information requests and descriptors of the Human Phenotype Ontology (HPO), directly linked to hereditary diseases. The resulting distance can be used to generate a probabilistic model, which can rank candidate diseases. Compared to termmatching approaches, the proposed approach performs better, especially for queries containing phenotypic noise or imprecise clinical descriptions. The semantic hierarchy defined by the HPO can be used to further refine the list if differential diagnosis. It is concluded that ontological distances can represent a useful approach to help physician to perform diagnosis procedures. 